\title{
Antimicrobial prescription behavior in 16 German intensive care units: room for improvement in pneumonia therapy
}

\author{
S Schneider ${ }^{1 *}$, J Zweigner ${ }^{2}$, F Schwab 1 , M Behnke ${ }^{1}$, E Meyer $^{3}$, P Gastmeier ${ }^{1}$ \\ From 3rd International Conference on Prevention and Infection Control (ICPIC 2015) \\ Geneva, Switzerland. 16-19 June 2015
}

\section{Introduction}

Antimicrobial surveillance in German hospitals is mainly based on consumption monitoring. Sparse data exist on prescription culture.

\section{Objectives}

To investigate the performance of antimicrobial prescription behavior in order to identify starting points for interventions.

\section{Methods}

16 intensive care units (ICUs) non-university hospitals covering Germany were included. Randomly selected medical records from patients diagnosed with pneumonia in 2012 were retrospectively analyzed. Bacteriological sampling and antimicrobial therapy (AMT) were reviewed with regard to clinically relevant aspects. Immunosuppressed, pregnant and underage patients were excluded.

\section{Results}

383 medical records were analyzed (179 community acquired and 204 hospital acquired pneumonia cases). $40.5 \%$ of empiric therapy regimens were appropriate according to national guidelines. Regimens with too broad spectrum or not recommended substances were used in $11.7 \% .47 .8 \%$ had formally too narrow spectra. Duration of therapy could only be evaluated in $40.7 \%$ of cases since the other patients were dimissed or died before recommended end of therapy. Within the evaluable group $31.4 \%$ of therapies were too long. Dosing was adequate in $86.6 \%$ of cases. Bacteriological sampling was performed as recommended in $23.1 \%$ of cases and

${ }^{1}$ Hygiene Institute, Charité, Berlin, Germany

Full list of author information is available at the end of the article incompletely in $51.2 \%$. Performance frequencies were $49.4 \%$ for blood cultures, $41.8 \%$ for tracheal aspirate and $15.4 \%$ for bronchoalveolar lavage. Legionella antigen was tested in only $7.6 \%$. De-escalation strategies could be evaluated in $76.0 \%$ of cases, since $24.0 \%$ were dismissed before day 4 . In $90 \%$ of evaluable cases no de-escalation was performed. In $6.9 \%$ de-escalation was microbiologically guided, in $3.1 \%$ clinically guided. Documentation of the indication for AMT was not performed in $25.5 \%$. In $61.9 \%$ the indication was placed unsystematically in the medical record. Documentation at a predefined place was only found in $12.6 \%$.

\section{Conclusion}

Large room for improvement in AMT was detected regarding duration of therapy, performance of bacteriological sampling, de-escalation concepts and documentation. The high percentage of formally too narrow empiric AMT regimens should be interpreted carefully. Further analysis is needed to determine the best performance indicators.

\section{Disclosure of interest}

None declared.

\section{Authors' details}

${ }^{1}$ Hygiene Institute, Charité, Berlin, Germany. ${ }^{2}$ Microbiology, Immunology, Hygiene, University Hospital, Cologne, Germany. ${ }^{3}$ Department of Hygiene, Städtisches Klinikum, Munich, Germany.

Published: 16 June 2015

doi:10.1186/2047-2994-4-S1-O2

Cite this article as: Schneider et al:: Antimicrobial prescription behavior in 16 German intensive care units: room for improvement in pneumonia therapy. Antimicrobial Resistance and Infection Control 2015 4(Suppl 1):O2. 